\title{
Isoperimetric Pentagonal Tilings
}

\author{
Ping Ngai Chung, Miguel A. Fernandez, Yifei Li, Michael Mara, \\ Frank Morgan, Isamar Rosa Plata, Niralee Shah, \\ Luis Sordo Vieira, Elena Wikner
}

T n 2001 Thomas Hales ([H]; see [M1, Chap. 15]) proved the Honeycomb Conjecture, which says that regular hexagons provide a leastperimeter tiling of the plane by unit-area regions. In this paper we seek perimeterminimizing tilings of the plane by unit-area pentagons. The regular pentagon has the least perimeter, but it does not tile the plane. There are many planar tilings by a single irregular pentagon or by many different unit-area pentagons; for some simple examples see Figure 3. Which of them has the least average perimeter per tile? Our main theorem, Theorem 3.5, proves that the Cairo and Prismatic tilings of Figure 1 minimize perimeter, assuming that the pentagons are convex. We conjecture that this convexity assumption

Ping Ngai Chung is a mathematics major at MIT. His email address is briancpn@mit.edu.

Miguel A. Fernandez is a mathematics major at Truman State University. His email address is maf2831@ truman. edu.

Yifei Li is a mathematics major at Berea College. Her email address is Yifei1124@gmai1.com.

Michael Mara is a mathematics major at Williams College. His email address is mtm1@wi $11 \mathrm{i}$ ams . edu.

Frank Morgan is professor of mathematics at Williams College. His email address is Frank. Morgan@wi 11 iams. edu.

Isamar Rosa Plata is a mathematics major at the University of Puerto Rico at Mayagüez. Her email address is silver. rose. isa@gmai1. com.

Niralee Shah is a mathematics major at Williams College. Her email address is nks1@wi $11 \mathrm{i}$ ams . edu.

Luis Sordo Vieira is a mathematics major at Wayne State University. His email address is dw8603@wayne. edu.

Elena Wikner is a recent graduate of Williams College. Her email address is elena.wikner@gmai 1.com.

DOI: http://dx.doi.org/10.1090/noti838 is unnecessary. The Cairo and Prismatic tiles have identical perimeter $2 \sqrt{2+\sqrt{3}} \approx 3.86$, less than a unit square's (4) but more than the regular pentagon's $(\approx 3.81)$. There are infinitely many equally efficient tilings by mixtures of Cairo and Prismatic tiles, as in Figure 2 (see also Remark 2.3 and Figures 5-16).

Tilings of the plane by pentagons have intrigued mathematicians and have been the subject of numerous studies (see the beautiful survey by Schattschneider [S3] and recent work of Sugimoto and Ogawa [SO]). Only in 1985 $[\mathrm{HH}]$ were the five types of equilateral convex pentagons that tile the plane proved complete. There are fourteen known types of convex pentagons that tile the plane, pictured in Figure 3 , and more tilings by nonconvex pentagons and mixtures of pentagons. For some appearances of pentagons throughout the world, see http://sites. wi 11iams. edu/Morgan/? $p=802$.

Along the way we prove an isoperimetric result we were not able to find in the literature (see Proposition 3.1 and Figure 17):

For $n$ given angles $0<a_{i} \leq \pi$ summing to ( $n-$ 2) $\pi$, the $n$-gon circumscribed about the unit circle maximizes area for given perimeter.

This complements the well-known result that, for given edge lengths, the $n$-gon inscribed in a circle maximizes area. (Idea of short proof: otherwise take the inscribed $n$-gon, with the surmounted circular arcs of the circle, and deform it to enclose more area. With the surmounted circular arcs, it now beats the circle, a contradiction.)

The proof that the Cairo and Prismatic tilings minimize perimeter begins with the Euler 


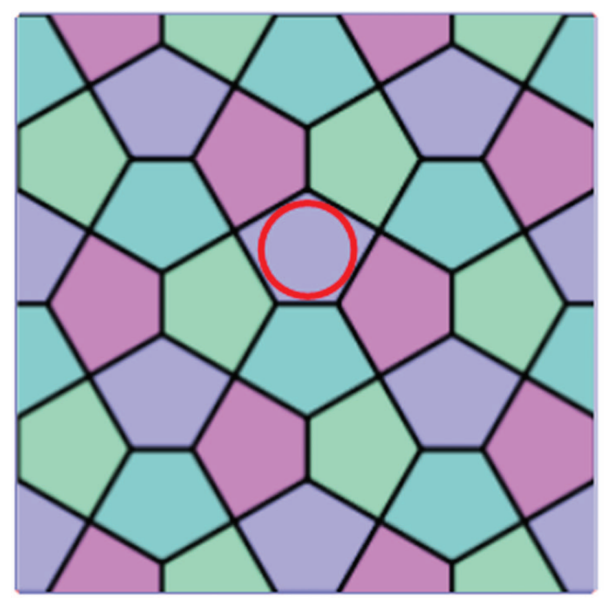

Cairo Pentagonal Tiling

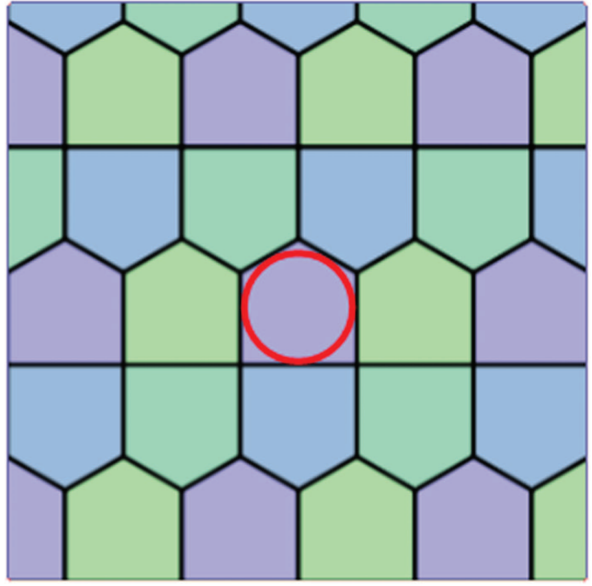

Prismatic Pentagonal Tiling

Figure 1. Minimal convex pentagonal tilings. Each tile has two right angles and three angles of $2 \pi / 3$ and is circumscribed about a circle.

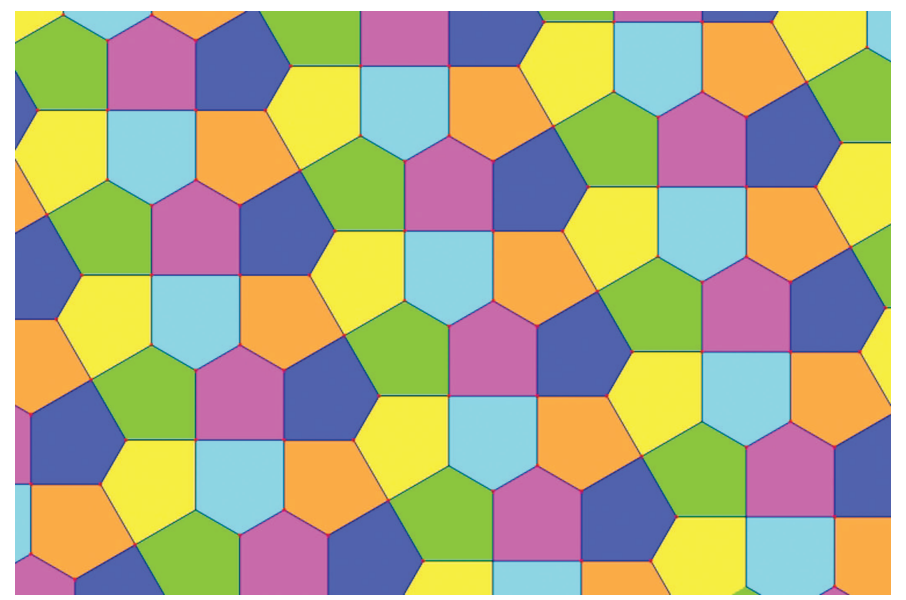

Figure 2. One of infinitely many equally efficient tilings by mixtures of Cairo and Prismatic pentagons.

characteristic formula, which implies that on average at least three angles per pentagon belong to vertices of the tiling of degree three. Thus at least $3 / 5$ of the angles are "large" angles with average measure $2 \pi / 3$. Certain convexity arguments show it best for three angles of each pentagon to equal $2 \pi / 3$ and for the others to equal $\pi / 2$, yielding the Cairo and Prismatic tiles. In the problematic case that one or two of the angles is $\pi$, the pentagon becomes a quadrilateral or a triangle and we need to enlarge our category to convex $n$-gons with $n \leq 5$, not necessarily meeting edge to edge (along entire edges). The Euler consequence generalizes to the statement that, on average, the number of large angles is at least $3 n-12$. Lower bounds for the perimeter contributed separately by triangles, quadrilaterals, and pentagons are compared via linear approximations.

\section{Tilings}

2.1. Definitions. A polygonal tiling is a decomposition of the plane as a union of polygonal regions which meet only along their boundaries. The tiling is edge to edge if the tiles meet only along entire edges. A monohedral tiling is a tiling by congruent copies of a single prototile. We do not restrict ourselves to monohedral tilings.

The perimeter ratio of a planar tiling is defined as the limit superior as $R$ approaches infinity of the perimeter inside an $R$-disc about the origin divided by the area $\pi R^{2}$, counting all edges and portions of edges inside the $R$-disc as in Figure 4 .

Although the terms are sometimes used more broadly, we define the Cairo and Prismatic pentagons as unit-area pentagons circumscribed about a circle with two right angles and three angles of $2 \pi / 3$, as in Figure 1. Proposition 3.1 


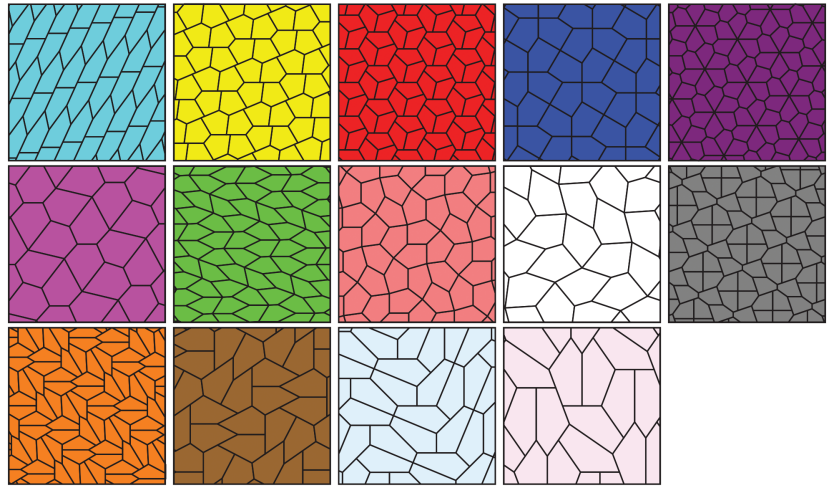

Figure 3. The fourteen known types of convex pentagons that tile the plane. http://en . wikipedia.org/wiki/Pentagon_tiling.

shows that circumscribed polygons minimize perimeter for given angles. The Cairo tile has one shorter edge of length $a=(2 / 3) \sqrt{6-3 \sqrt{3}} \approx$ .5977 and four equal longer edges of length $b=(3+\sqrt{3}) \sqrt{2-\sqrt{3}} / 3 \approx .8165$. The Prismatic tile has two adjacent shorter edges of length $a$ (forming the roof of the house), two edges of length $b$ (the walls), and a still longer base of length $c=2 \sqrt{2-\sqrt{3}} \approx 1.0353$.

Proposition 2.2. There is a unique planar tiling by Cairo tiles. There is a unique edge-to-edge planar tiling by Prismatic tiles.

We call these the Cairo and Prismatic tilings (pictured in Figure 1).

Proof. For the Cairo tiles, the shorter edges have vertices of degree 3 , so every tiling must be edge to edge. Working out from a vertex of degree 4 , it is easy to see that an edge-to-edge tiling by the Cairo or the Prismatic tile is unique.

Remark 2.3 (Nonuniqueness). The first version of this paper asked whether a tiling of the plane by a mixture of Cairo and Prismatic tiles, which we now call a Cairo-Prismatic tiling, exists. During the application process for Frank Morgan's 2011 NSF "SMALL" undergraduate research Geometry Group, a freshman at MIT, shortly recruited as a coauthor (P. N. Chung), discovered uncountably many tilings by mixtures of Cairo and Prismatic tiles. In Figure 2, there are alternating diagonal rows of Cairo and Prismatic tiles. The Cairo tiles are grouped in fours into hexagons, while the Prismatic tiles are grouped in pairs. Uncountably many other such tilings may be obtained by placing arbitrarily many rows of one type before rows of the other type. Most of these tilings are nonperiodic. One other example is shown in Figure 5.
After writing this paper, we came across the older, nonperiodic example of Figure 6. It was discovered by the now famous housewife Marjorie Rice [R], featured in Doris Schattschneider's article "In Praise of Amateurs" [S1, Fig. 15]. Rice had no interest in minimizing perimeter; apparently she simply found it a beautiful tiling by two pentagonal prototiles. Our original goal had been to prove that the tilings shown in Figure 2 were the only tilings by mixtures of Cairo and Prismatic Pentagons. In attempting a proof and by trial and error with Geometer's Sketchpad, we found many more CairoPrismatic tilings, some with symmetries of four of the seventeen wallpaper groups (Figures 7-11) and others with fewer or no symmetries (Figures $12-16) ;$ see $[C]$ for more. We wonder whether there are examples for the other wallpaper groups. For a chart of the seventeen wallpaper groups and their respective symmetries, see [S2] or [W].

Daniel Huson [Hu] has found tilings by many other pairs of prototiles.

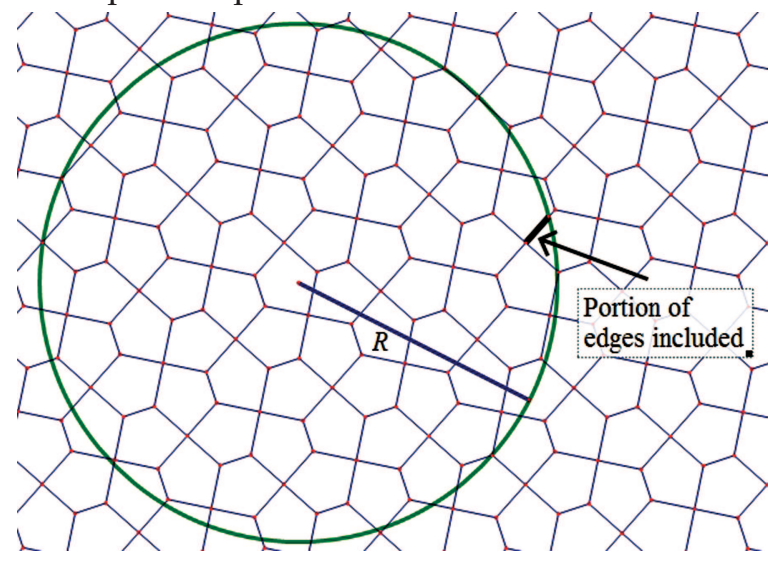

Figure 4. The perimeter ratio is the limit superior as $R$ approaches infinity of the perimeter inside an $\boldsymbol{R}$-disc, including portions of edges, divided by the area $\pi R^{2}$.

\section{Isoperimetric Pentagonal Tilings}

Our main theorem, Theorem 3.5, proves that the Cairo and Prismatic tilings minimize perimeter among all tilings by unit-area convex polygons with at most five sides. We begin with the isoperimetric Proposition 3.1, which we have not been able to find in the literature, perhaps because it depends on knowledge of the ideal "Wulff shape" for crystals [M2, 10.6]. Note that, for given ordered angles or equivalently given orientation of the edges, there is a unique $n$-gon as in Figure 17 circumscribed about the unit circle by just taking the edges tangent to the circle; this is the $n$-gon which minimizes perimeter for given area.

Proposition 3.1. For $n$ given angles $0<a_{i} \leq \pi$ summing to $(n-2) \pi$, the $n$-gon circumscribed 


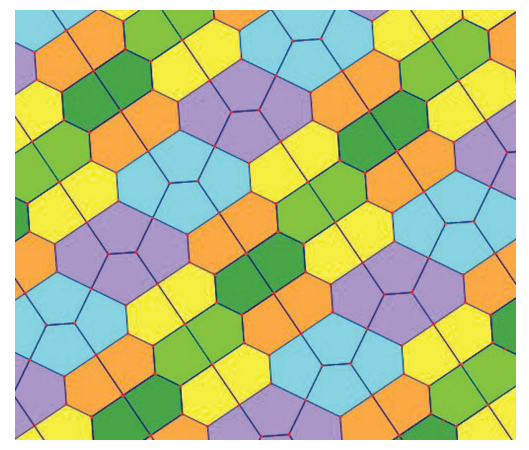

Figure 5. Another tiling by a mixture of Cairo and Prismatic tiles, in which the blue-purple diagonal rows of Cairo tiles are separated by three diagonal rows of Prismatic tiles.

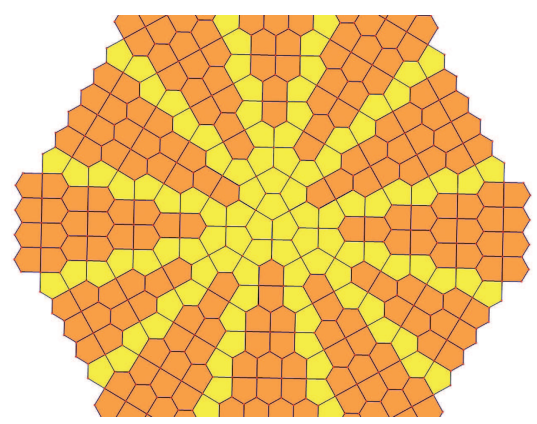

Figure 6. A nonperiodic tiling by a mixture of Cairo and Prismatic tiles due to Marjorie Rice ([R] or [S1, Fig. 15]).

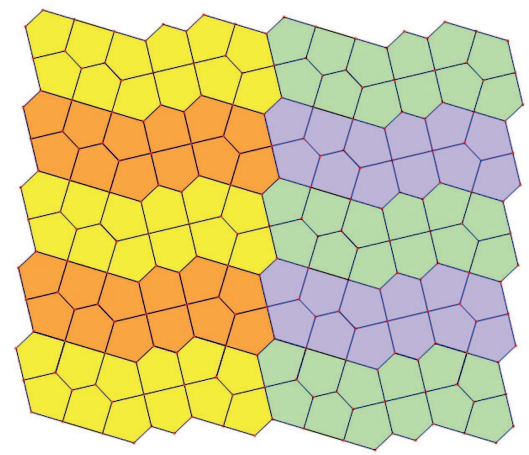

Figure 7. "Sardines" Cairo-Prismatic tiling with wallpaper group $p 1$.

about the unit circle as in Figure 17 is uniquely perimeter minimizing for its area. Scaled to unit area, its perimeter is

$$
2 \sqrt{\cot \left(a_{i} / 2\right)} .
$$

Since cotangent is strictly convex up to $\pi / 2$, the more nearly equal the angles, the smaller the perimeter.

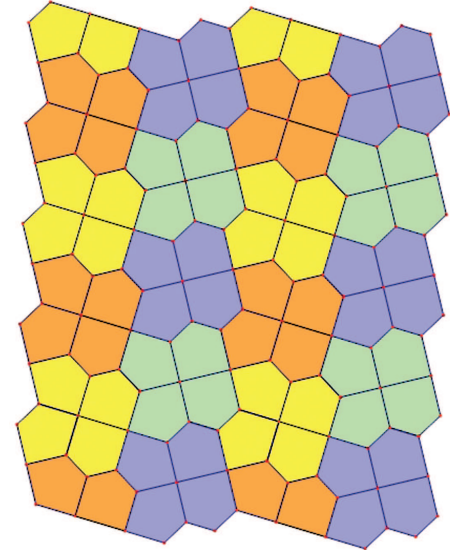

Figure 8. "Stripes" Cairo-Prismatic tiling with wallpaper group $p 2$.

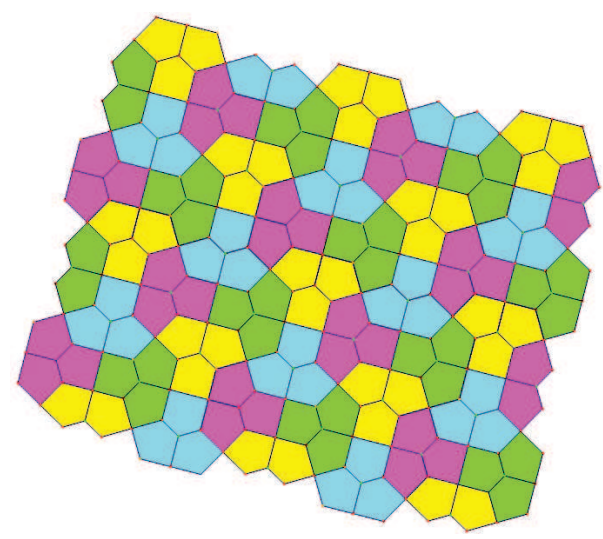

Figure 9. "Pills" Cairo-Prismatic tiling with wallpaper group $p 4 g$.

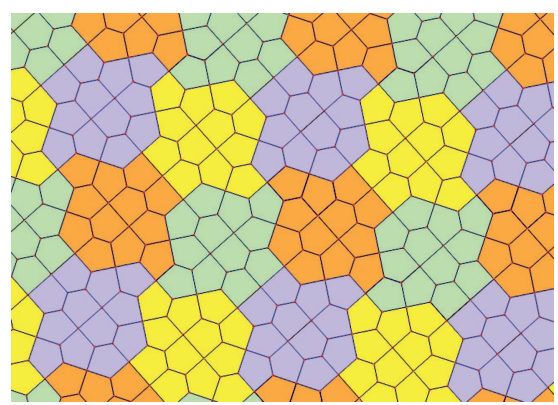

Figure 10. "Spaceship" Cairo-Prismatic tiling with wallpaper group $p 4 g$.

Proof. (See [M2, 10.6].) The celebrated isoperimetric theorem says that, for the Euclidean norm on $\mathbf{R}^{2}$ (or $\mathbf{R}^{n}$ ), balls provide the least-perimeter way to enclose given area (or volume). There is a less familiar generalization to other (not necessarily even) norms $\Phi$, under which the length of a curve is weighted by $\Phi$ applied to the unit normal. Then the least-perimeter way to enclose given area is 


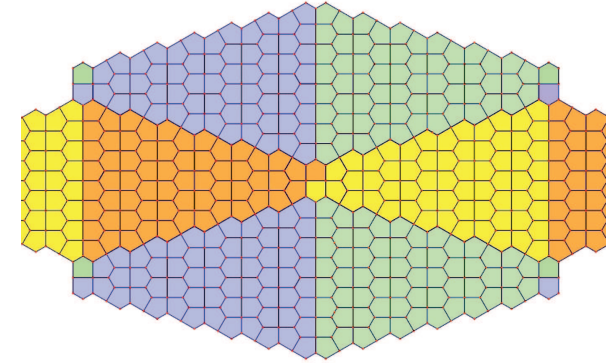

Figure 11. "Christmas Tree" Cairo-Prismatic tiling with wallpaper group $\mathrm{cmm}$.

given by scalings of the "Wulff shape" or unit ball in the dual norm $\Phi^{*}$, defined by

$$
\Phi^{*}(x)=\sup \{x \cdot y: \Phi(y) \leq 1\} .
$$

In particular, a polygon $P$ circumscribed about the unit circle is the unit $\Phi^{*}$ ball, where the unit $\Phi$ ball is the inscribed polygon with vertices at the points of tangency of $P$. Note that $\Phi$ is 1 on the sides of $P$ and some irrelevant larger value in other directions.

Therefore $P$ minimizes Euclidean length for the given orientations of its sides, as desired. By simple geometry, its perimeter $P_{0}$ and area $A_{0}$ satisfy

$$
P_{0}=2 A_{0}=2 \sum \cot \left(a_{i} / 2\right) .
$$

The final formula for unit area follows by scaling.

Remarks. If you allow angles between $\pi$ and $2 \pi$, the circumscribed polygon still minimizes perimeter for sides in the associated directions, but they may necessarily occur in a different order and produce different angles and a smaller perimeter. If you require the prescribed order to keep the prescribed angles, some sides may become infinitesimally small, thus omitted, replacing pairs of external angles with their sum and reducing our equation (1). For $n>3$, the least-perimeter unit-area $n$-gon with one small prescribed angle is not convex; for $n=4$ it is close to being an equilateral triangle with a small nonconvex protrusion near one of the three vertices, as in Figure 18.

The proof of Theorem 3.5, which shows that certain features of tiles which hold on average ideally hold exactly, depends on the following convexity arguments, summarized in Corollary 3.4. By Proposition 3.1, the $f(u)$ of Lemma 3.2 is proportional to the perimeter of a unit-area $n$-gon with $k$ angles of $2 \theta$ and $k^{\prime}$ angles of $2 \theta^{\prime}$. Convexity means that the closer together these angle sizes, the better. Lemma 3.3 allows $k$ and $k^{\prime}$ to vary and shows furthermore that ideally all $n$-gons have the same number $k$ of large angles.

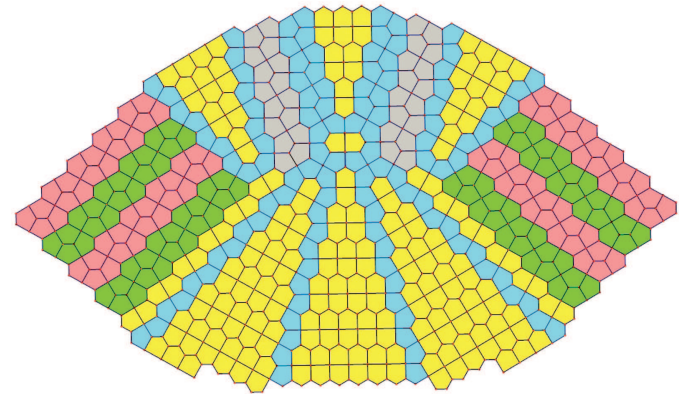

Figure 12. "Bunny" Cairo-Prismatic tiling with symmetry group $D_{1}$.

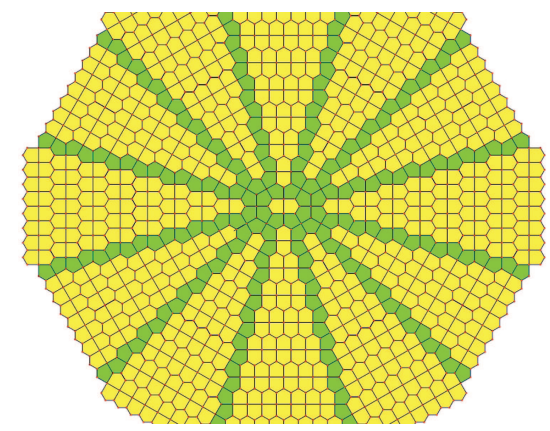

Figure 13. "Plaza" Cairo-Prismatic tiling with symmetry group $D_{2}$.

Lemma 3.2. For positive integers $k+k^{\prime}=n$, consider the positive function

$$
f^{2}(u)=g(u)=k t+k^{\prime} t^{\prime}
$$

where $t=\cot \theta, t^{\prime}=\cot \theta^{\prime}, 0<\theta, \theta^{\prime}<\pi / 2$, $\theta=k^{\prime} u, \theta^{\prime}=(n-2) \pi / 2 k^{\prime}-k u$. Then $f$ is strictly convex.

Proof. It suffices to show that $2 g g^{\prime \prime}-g^{\prime 2}$ is positive (where primes on $g$ denote differentiation with respect to $u$ ). Writing $s=\csc \theta, s^{\prime}=\csc \theta^{\prime}$, we compute that

$$
\begin{aligned}
g^{\prime} & =-k k^{\prime} s^{2}+k k^{\prime} s^{2}, \\
g^{\prime \prime} & =2 k k^{\prime 2} s^{2} t+2 k^{2} k^{\prime} s^{\prime 2} t^{\prime},
\end{aligned}
$$

and after discarding some positive terms

$$
\begin{aligned}
2 g g^{\prime \prime}-g^{\prime 2}> & 2 k^{2} k^{\prime 2} s^{2} t^{2}+2 k^{2} k^{\prime 2} s^{\prime 2} t^{\prime 2} \\
& -k^{2} k^{\prime 2} s^{4}-k^{2} k^{\prime 2} s^{\prime 4}+2 k^{2} k^{\prime 2} s^{2} s^{\prime 2},
\end{aligned}
$$

which is proportional to

$$
\begin{aligned}
2 s^{2} t^{2}+ & 2 s^{\prime 2} t^{\prime 2}-s^{4}-s^{\prime 4}+2 s^{2} s^{\prime 2} \\
& >2 s^{2} t^{2}+2 s^{\prime 2} t^{\prime 2}-s^{4}-s^{\prime 4}+s^{2}+s^{\prime 2} \\
& =2 s^{2} t^{2}+2 s^{\prime 2} t^{\prime 2}-s^{2} t^{2}-s^{\prime 2} t^{\prime 2}>0 .
\end{aligned}
$$

Lemma 3.3. For positive constants $n, c, c^{\prime}$ and nonnegative constants $d, d^{\prime}$, consider the positive function

$$
f^{2}(k)=g(k)=k t+k^{\prime} t^{\prime},
$$




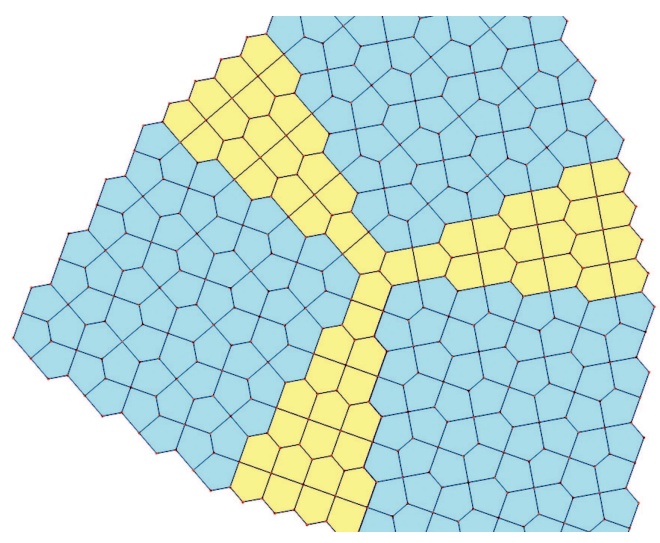

Figure 14. "Waterwheel" Cairo-Prismatic tiling with symmetry group $D_{3}$.

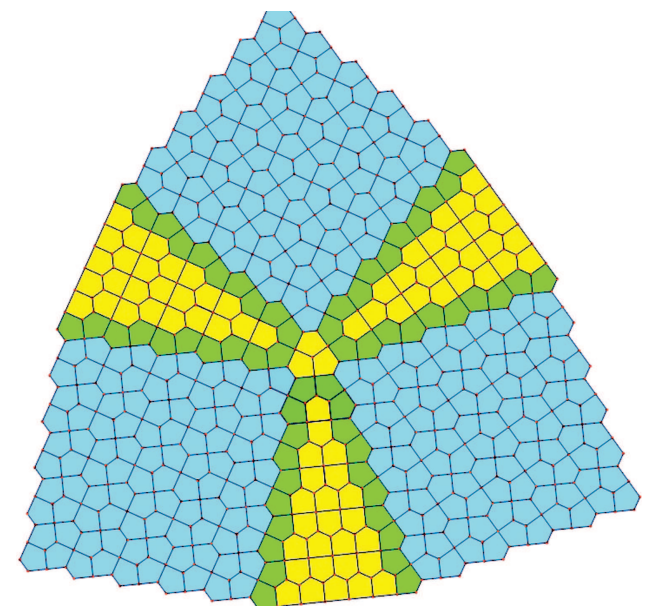

Figure 15. "Windmill" Cairo-Prismatic tiling with symmetry group $D_{3}$.

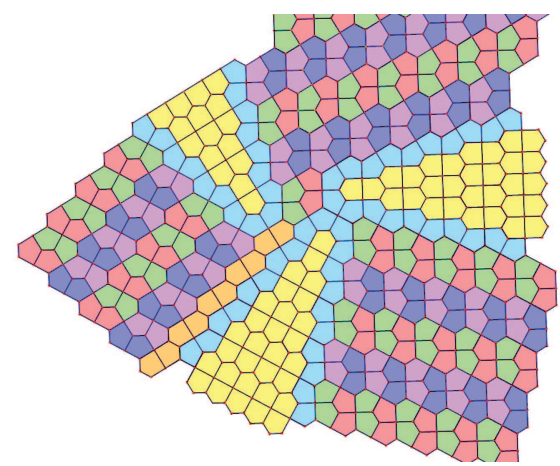

Figure 16. "Chaos” Cairo-Prismatic tiling with no symmetry.

where $0<k<n, k^{\prime}=n-k, t=\cot \theta, t^{\prime}=\cot \theta^{\prime}$, $0<\theta, \theta^{\prime}<\pi / 2, \theta=d-c / k, \theta^{\prime}=d^{\prime}-c^{\prime} / k^{\prime}$, and $\min \left\{\theta, \theta^{\prime}\right\} \leq \pi / 3$. Then $f$ is strictly convex.

Proof. It suffices to show that $2 g g^{\prime \prime}-g^{\prime 2}$ is positive. Write $s=\csc \theta, s^{\prime}=\csc \theta^{\prime}$. By symmetry we

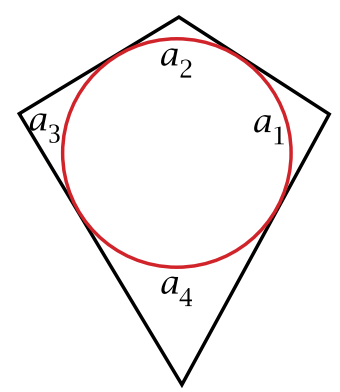

Figure 17. For given angles the least-perimeter unit-area polygon is circumscribed about a circle.

may assume that $\theta \leq \theta^{\prime}$, so $c / k \geq c^{\prime} / k^{\prime}, t \geq t^{\prime}$, and $s \geq s^{\prime}$. We compute that

$$
\begin{aligned}
g^{\prime} & =t-k s^{2}\left(c / k^{2}\right)-t^{\prime}+k^{\prime} s^{\prime 2}\left(c^{\prime} / k^{\prime 2}\right) \\
& =t-t^{\prime}-\left(s^{2} c / k-s^{\prime 2} c^{\prime} / k^{\prime}\right)
\end{aligned}
$$

so

$$
-s^{2} c / k \leq g^{\prime} \leq t-t^{\prime}
$$

Since

$$
t-t^{\prime} \leq s^{2}\left(\theta^{\prime}-\theta\right) \leq s^{2} c / k,
$$

we see that

$$
\left|g^{\prime}\right| \leq s^{2} c / k
$$

We further compute that

$$
g^{\prime \prime}=2 s^{2} t c^{2} / k^{3}+2 s^{\prime 2} t c^{\prime 2} / k^{\prime 3} .
$$

Hence

$$
2 g g^{\prime \prime}-g^{\prime 2}>2(k t)\left(2 s^{2} t c^{2} / k^{3}\right)-s^{4} c^{2} / k^{2},
$$

which is proportional to $4 t^{2}-s^{2}$, which is nonnegative because $\theta \leq \pi / 3$.

Corollary 3.4. For integers $n \geq 3$, the positive function $f_{n}$ defined by

$$
f_{n}^{2}(k, k \theta)=k \cot \theta+k^{\prime} \cot \theta^{\prime},
$$

where $k^{\prime}$ and $\theta^{\prime}$ are defined by $k+k^{\prime}=n, k \theta+$ $k^{\prime} \theta^{\prime}=(n-2) \pi / 2,0<k, k^{\prime}<n, 0<\theta, \theta^{\prime}<\pi / 2$, and $\min \left\{\theta, \theta^{\prime}\right\} \leq \pi / 3$ is strictly convex.

Proof. On a vertical line in the domain ( $k$ constant), $f_{n}$ is strictly convex by Lemma 3.2. On a nonvertical line, $f_{n}$ is strictly convex by Lemma 3.3.

Theorem 3.5. Perimeter-minimizing planar tilings by unit-area convex polygons with at most five sides are given by Cairo and Prismatic tiles (as in Figures 1, 2, 5-16).

Remarks. There is never uniqueness, since compact variations as in Figure 19 do not change the limiting perimeter ratio.

For doubly periodic or monohedral tilings, however, there are no others, although the Prismatic tilings need not be edge to edge.

If nonconvex tiles are allowed, the result remains conjectural (except for monohedral tilings, 


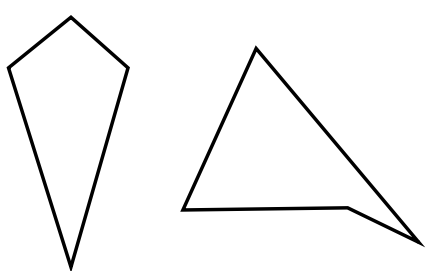

Figure 18. The least-perimeter unit-area quadrilateral with one small prescribed angle is not the convex quadrilateral with the other angles equal but a triangle with a small nonconvex protrusion.

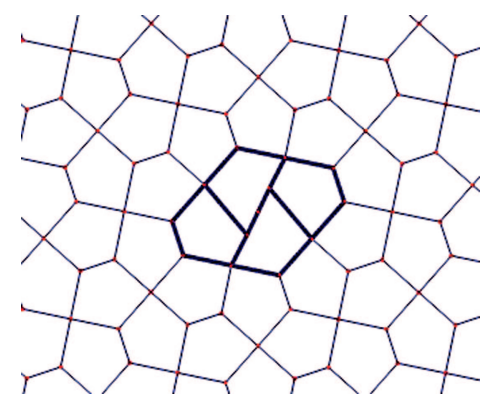

Figure 19. An example of a compact variation of the Cairo tiling, which remains perimeter minimizing.

when such a large angle gives the prototile more perimeter than a unit square).

We are not assuming that the tiling is monohedral or edge to edge.

The proof of Theorem 3.5 reduces the question about tilings to a question about abstract finite collections of polygons satisfying certain conditions (Step III). In Steps IV and V, Corollary 3.4 is applied to get a sharp lower bound on the perimeter, with strict inequality for triangles and 4-gons and equality for Cairo and Prismatic pentagons. The proof begins with careful truncation to reduce the question to collections of $N$ polygons with nice limit properties as $N$ approaches infinity.

Proof of Theorem 3.5. Consider a planar tiling with bounded perimeter ratio $\rho$ by unit-area convex polygons with at most five sides. We may assume that every angle is strictly less than $\pi$ by eliminating vertices at angles of $\pi$ from polygons. (Recall that we are not assuming that the tilings are edge to edge.)

Step I: Truncation. To control truncation we claim that, for some sequence of $R$ going to infinity, the circle about the origin of radius $R$ meets the tiling in $o\left(R^{2}\right)$ points (i.e., the ratio to $R^{2}$ goes to 0 ). Otherwise, for almost all large $R$, for some $\varepsilon>0$, since $P$ grows at a rate at least equal to the number of such points (see [M1, 15.3] for details),

$$
d P / d r \geq \varepsilon R^{2} \geq(\varepsilon / \rho) P .
$$

This implies that perimeter and hence area grow exponentially. Since area equals $\pi R^{2}$, this is a contradiction.

For such a sequence of $R$, consider the tiles inside the disc $D_{R}$ of radius $R$. The ratio of their area to $\pi R^{2}$ approaches 1 , while the number of adjacent discarded tiles is $o\left(R^{2}\right)$, which is negligible. Therefore the perimeter ratio inside $D_{R}$ equals the average cost of the tiles inside $D_{R}$ up to $o(1)$.

Step II: The fraction $f_{n}$ of polygons with $n$ edges, the number $e$ of edges per polygon, and the number of vertices $v_{d}$ per polygon of degree $d$. We will examine more carefully our sequence as $R$ approaches infinity. By taking a subsequence if necessary, we may assume that the fraction of polygons of $n$ edges approaches a limit $f_{n}$, hence that the number of edges per polygon approaches $e=\sum n f_{n}$, and that the number of vertices per polygon of degree $d$ in the tiling approaches a limit $v_{d}$. Thus

$$
\sum v_{d}=e
$$

By Euler, for the tiles inside $D_{R}$,

(2) $V=E-F+1 \geq e F / 2-F+1=(e / 2-1) F+1$,

with equality up to $o(1)$ if the tiling is edge to edge. Hence, if the tiling is edge to edge, the following equality holds up to $o(1)$ for the tiles inside $D_{R}$ :

$$
\sum v_{d} / d=V / F=e / 2-1 \text {. }
$$

If the tiling is not edge to edge, we count a vertex where $k-1$ other edges meet in the interior of an edge as contributing to $v_{2 k}$ instead of $v_{k}$, because these polygon angles are $2 \pi / 2 k$ on the average. Such a vertex counts just $1 / 2$ on the left side of (3). Let $V^{*}$ be the number of such vertices. Then

(4)

$$
\begin{aligned}
\sum v_{d} / d & =\left(V-V^{*} / 2\right) / F=\left(E-V^{*} / 2-F\right) / F \\
& =\left(\left(e F+V^{*}\right) / 2-V^{*} / 2-F\right) / F=e / 2-1,
\end{aligned}
$$

because each such vertex breaks a polygonal edge into two edges, replacing $e F$ with $e F+V^{*}$. In the limit, equation (4) holds exactly. By (1) and (4),

$$
\begin{aligned}
& v_{3} / 3+\left(e-v_{3}\right) / 4 \geq e / 2-1, \\
& v_{3} \geq 3 e-12 .
\end{aligned}
$$

Step III: The reduction from tilings to abstract collections of polygons with $k_{n}$ large angles averaging $2 \theta_{n}$. Now it suffices to prove that, for every collection of $N$ convex unit-area polygons with at most five edges with $(3 e-12) N-N_{1}$ angles of average measure at least $2 \pi / 3$ with $N_{1}=o(N)$, the average perimeter is at least nearly that of the 
Cairo and Prismatic pentagons. Among all such collections of $N$ pentagons, choose one to minimize the average perimeter. By Proposition 3.1, they are all circumscribed about circles. Since, by Proposition 3.1, reducing large angles reduces perimeter, the $(3 e-12) N-N_{1}$ "large" angles have average measure exactly $2 \pi / 3$ and are all at least as large as the remaining "small angles", all at most $2 \pi / 3$. Up to $o(N)$, the number of small angles is $(12-2 e) N$, their sum is $(6-e) \pi N$, so their average is $\pi / 2+o(1)$, and all large angles are at least that large.

For each $3 \leq n \leq 5$, we may assume that the average large angle in an $n$-gon approaches a limit $2 \theta_{n}$ and that the number of large angles per $n$-gon approaches a limit $k_{n}$. Then

(6)

$$
\sum f_{n} k_{n}=3 e-12, \quad \sum f_{n} k_{n} \theta_{n}=(\pi / 3) \sum f_{n} k_{n} .
$$

Since $e=\sum n f_{n}$, we see that

$$
\begin{aligned}
& f_{3}\left(k_{3}+3\right)+f_{4} k_{4}+f_{5}\left(k_{5}-3\right)=0, \\
& f_{3}\left(k_{3} \theta_{3}+\pi\right)+f_{4} k_{4} \theta_{4}+f_{5}\left(k_{5} \theta_{5}-\pi\right)=0 .
\end{aligned}
$$

Note that each quadrilateral has at least one large angle or it could be replaced by a Cairo pentagon, increasing both $(3 e-12) N-N_{1}$ and the number of large angles by three and decreasing perimeter.

By Proposition 3.1, in each $n$-gon the large angles are all equal and the small angles are all equal.

Step IV: The consequences of convexity. By convexity (Corollary 3.4), for each $3 \leq n \leq 5$, in the limit the average perimeter is bounded below by the values of the perimeter function $P_{n}(k, q)$ at $k_{n}$ and $k_{n} \theta_{n}$, where

$$
P_{n}(k, q)=2\left(k \cot q / k+k^{\prime} \cot q^{\prime} / k^{\prime}\right)^{1 / 2},
$$

$k+k^{\prime}=n, q+q^{\prime}=(n-2) \pi / 2$. This function corresponds to the perimeter of an $n$-gon with $k$ large angles, each of measure $2 q / k$. In particular, $P_{5}(3, \pi)$ corresponds to the perimeter of a Cairo or Prismatic pentagon. We compute to four decimal places that

$$
\begin{aligned}
& \left(\partial P_{3} / \partial k\right)(1 / 2, \pi / 8) \approx-.7217, \\
& \left(\partial P_{3} / \partial q\right)(1 / 2, \pi / 8) \approx 1.2265, \\
& \left(\partial P_{4} / \partial k\right)(1, \pi / 3) \approx-.4455, \\
& \left(\partial P_{4} / \partial q\right)(1, \pi / 3) \approx .5334 \\
& \left(\partial P_{5} / \partial k\right)(3, \pi) \approx-.3091, \\
& \left(\partial P_{5} / \partial q\right)(3, \pi) \approx .3451 .
\end{aligned}
$$

Note that $f_{5}>0$; otherwise, by (7) $f_{4}=1, k_{4}=0$, and the square tiling provides a lower bound, while we know that the Cairo tiling is better. Also by $(7), k_{5} \leq 3$. Since the average small angle is $\pi / 2$, every large angle $2 \theta_{n}$ is at least $\pi / 2, k_{3}<2$, and $k_{4}<3$. Since the sum of the angles of an $n$-gon is $(n-2) \pi, k_{n} \theta_{n}+k_{n}^{\prime} \theta_{n}^{\prime}=(n-2) \pi / 2, \theta_{5} \geq 3 \pi / 10$ and $\theta_{n}^{\prime} \leq(n-2) \pi / 2 n$.

Step V: The lower bound on perimeter. Since, by Corollary 3.4 , each $f_{n}$ is convex, we can bound the perimeter $P$ per tile from below by the linear approximations:

$$
\begin{gathered}
P=f_{3} P_{3}\left(k_{3}, k_{3} \theta_{3}\right)+f_{4} P_{4}\left(k_{4}, k_{4} \theta_{4}\right)+f_{5} P_{5}\left(k_{5}, k_{5} \theta_{5}\right) \\
\geq f_{3}\left[P_{3}(1 / 2, \pi / 8)+\left(\partial P_{3} / \partial k\right)(1 / 2, \pi / 8)\left(k_{3}-1 / 2\right)\right. \\
\left.+\left(\partial P_{3} / \partial q\right)(1 / 2, \pi / 8)\left(k_{3} \theta_{3}-\pi / 8\right)\right] \\
+f_{4}\left[P_{4}(1, \pi / 3)+\left(\partial P_{4} / \partial k\right)(1, \pi / 3)\left(k_{4}-1\right)\right. \\
\left.+\left(\partial P_{4} / \partial q\right)(1, \pi / 3)\left(k_{4} \theta_{4}-\pi / 3\right)\right] \\
+f_{5}\left[P_{5}(3, \pi)+\left(\partial P_{5} / \partial k\right)(3, \pi)\left(k_{5}-3\right)\right. \\
\left.+\left(\partial P_{5} / \partial q\right)(3, \pi)\left(k_{5} \theta_{5}-\pi\right)\right] .
\end{gathered}
$$

Using (7) to substitute for $f_{5}\left(k_{5}-3\right)$ and $f_{5}\left(k_{5} \theta_{5}-\right.$ $\pi)$, we obtain

$$
P \geq f_{5} P_{5}(3, \pi)+f_{3} Q_{3}+f_{4} Q_{4},
$$

where (rounding appropriately)

$$
\begin{aligned}
Q_{3}> & P_{3}(1 / 2, \pi / 8)+(1 / 2)(.7216) \\
& -(\pi / 8)(1.2266)+3(.3090)-\pi(.3452) \\
& +k_{3}(-.7218+.3090)+k_{3} \theta_{3}(1.2264-.3452) \\
> & 4.6503+(1 / 2)(.7216)-(\pi / 8)(1.2266) \\
& +3(.3090)-\pi(.3452) \\
& +k_{3}(-.7218+.3090)+k_{3} \theta_{3}(1.2264-.3452) \\
> & 4.3718+k_{3}(-.4128+(\pi / 4)(.8812)) \\
> & P_{5}(3, \pi)
\end{aligned}
$$

and

$$
\begin{aligned}
Q_{4}> & P_{4}(1, \pi / 3)+.4454-(\pi / 3)(.5335) \\
& +k_{4}(-.4456+.3090)+k_{4} \theta_{4}(.5333-.3452) \\
> & 3.9622+k_{4}(-.1366+(\pi / 4)(.1881)) \\
> & P_{5}(3, \pi) .
\end{aligned}
$$

Therefore the perimeter per tile is at least the perimeter $P_{5}(3, \pi)$ of the Cairo and the Prismatic tiles, as desired.

For doubly periodic tilings, the argument can be done on the torus without limits or truncation error, and every tile must be Cairo or Prismatic.

Remark. Theorem 3.5 holds for a monohedral edge-to-edge planar tiling by a curvilinear polygon $\mathrm{P}$ (embedded rectifiable curves meeting only at vertices) with at most five edges. Because the tiling is monohedral and edge-to-edge, one can replace $\mathrm{P}$ with an immersed rectilinear n-gon with the same vertices, as in Figure 20. If it is not embedded, it has more perimeter than a square.

There remains the opening conjecture that the Cairo and Prismatic tilings remain perimeter minimizing if one allows mixing in some nonconvex pentagonal tiles (along with lots of nearly regular pentagonal tiles). 

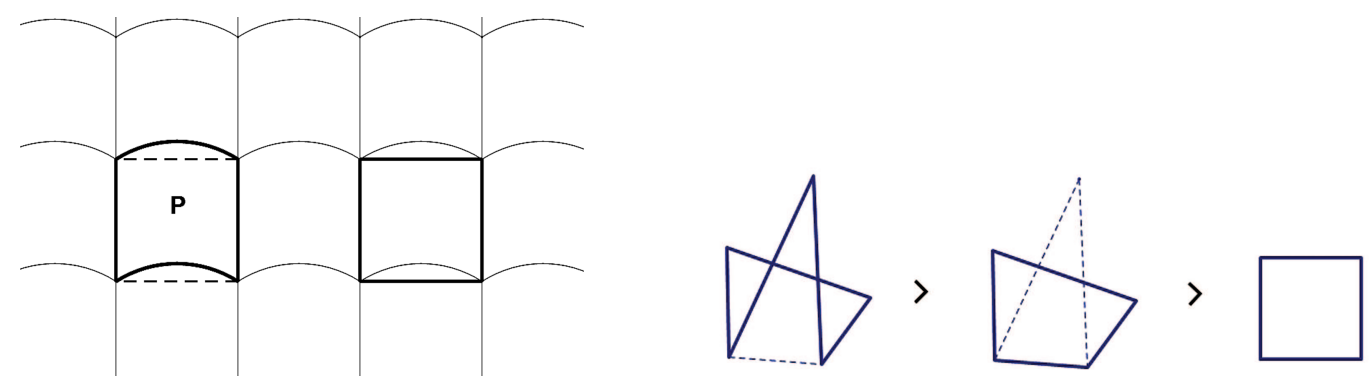

Figure 20. Each tile in a monohedral edge-to-edge planar tiling by a curvilinear polygon $\mathrm{P}$ can be replaced by an immersed rectilinear $n$-gon with the same vertices (left). If the $n$-gon is not embedded, it has more perimeter than a square (right).

\section{Acknowledgments}

We thank Doris Schattschneider, Daniel Huson, Max Engelstein, David Thompson, and an anonymous referee for their help. F. Morgan thanks the Fields Institute, where he spent fall 2010 on sabbatical. For funding we thank the National Science Foundation for grants to Morgan and to the Williams College "SMALL" REU, Williams College, Berea College, and the Mathematical Association of America for supporting trips to speak at MathFest.

\section{References}

[C] Ping Ngai Chung, Miguel A. Fernandez, Niralee SHAH, Luis SORdo VIEIRA, ElENA WIKNER, Perimeterminimizing pentagonal tilings, preprint (2011).

[H] Thomas C. Hales, The Honeycomb Conjecture, Disc. Comp. Geom. 25:1-22 (2001); available at http://arxiv.org/abs/math.MG/9906042/

[HH] M. D. HIRSCHHORN and D. C. HuNT, Equilateral convex pentagons which tile the plane, J. Comb. Theory A 39 (1985), 1-18.

[Hu] DANIEL HuSON, 2DTiler, http://ab.inf.unituebingen.de/software/2dtiler/welcome.htm7.

[L] YIFEI LI, MiCHAEL MARA, ISAMAR ROSA PlATA, and ELENA WIKNER, Tiling with penalties and isoperimetry with density, preprint (2010).

[M1] FRANK MORGAN, Geometric Measure Theory: A Beginner's Guide, 4th ed., Academic Press, 2009.

[M2] Liemannian Geometry: A Beginner's Guide, 2nd ed., A K Peters, Wellesley, 1998, revised printing, 2001.

[R] MARJORIE RICE, Intriguing tessellations, http:// home.comcast.net/ tesse11ations/tesse11ations. htm, accessed 23 June 2011.

[S1] DORIS SCHATTSCHNEIDER, In praise of amateurs, David Klarner, ed., The Mathematical Gardner, Prindle, Weber \& Schmidt, 1981, 140-166.

[S2] _ , The plane symmetry groups: Their recognition and notations, Amer. Math. Monthly 85 (6) (1978), 439-450.

[S3] _ , Tiling the plane with congruent pentagons, with Afterword, The Harmony of the World: 75 Years of Mathematics Magazine, Gerald Alexanderson and Peter Ross, eds., Math. Assn. Amer., 2007, 175-190.

[SO] Teruhisa Sugimoto and Tohru Ogawa, Properties of nodes in pentagonal tilings, Forma 24 (2009), 117-121; available at http://www. scipress.org/journa1s/forma/pdf/2403/24030117. pdf.

[W] Wikipedia Commons: Wallpaper group. Last modified 6 July 2011, accessed 14 July 2011. 